\title{
Indirekte traumatische Optikusneuropathie nach Fahrradsturz
}

\author{
Sarah Krainz · Mona Regina Schneider · Bianca Bizjak · Andreas Wedrich
}

Eingegangen: 6. April 2016 / Angenommen: 28. April 2016 / Online publiziert: 19. Mai 2016

(C) The Author(s) 2016. This article is available at SpringerLink with Open Access

\begin{abstract}
Zusammenfassung Wir berichten über einen 34-jährigen Patienten, der im Rahmen eines Fahrradsturzes eine Orbitafraktur erlitt und posttraumatisch keine Lichtwahrnehmung am betroffenen Auge aufwies. Funduskopisch zeigte sich eine subretinale peripapilläre Blutung im Sinne einer traumatischen Optikusneuropathie. Der Visus erholte sich bereits wenige Stunden posttraumatisch ohne spezifische Therapie und war am nächsten Tag bereits auf 0,4 angestiegen. Zwei Monate später zeigte sich ein Endvisus von 0,63 und die Papille imponierte partiell atrophisch. In den letzten Jahren wurden einige Studien und Fallserien zur hochdosierten Kortisontherapie bei traumatischer Optikusneuropathie publiziert. Es zeigte sich eine relativ hohe Rate an spontanen Visusverbesserungen und keine überzeugenden Daten, dass die Kortisontherapie der alleinigen Beobachtung überlegen wäre. Eine Übersicht der publizierten Artikel zeigt die kontroverse Diskussion im Hinblick auf hochdosierte Kortisontherapie. Unser Fallbericht bestätigt, dass spontane partielle Remissionen beobachtet werden.
\end{abstract}

Schlüsselwörter Sehnervenverletzungen - Traumatische Optikusneuropathie · Kortison · Optikusatrophie

\section{Indirect traumatic optic neuropathy after bicycle crash}

Summary We report on a 34-year-old male patient, who suffered from an orbital fracture and had no

Dr. med. univ. S. Krainz $(\bowtie) \cdot$ Dr. med. univ. M. R. Schneider • Dr. med. univ. B. Bizjak · Univ. Prof. Dr. med. univ. A. Wedrich Universitätsaugenklinik, Medizinische Universität Graz, Auenbruggerplatz 4, 8036 Graz, Österreich E-Mail: sarah.krainz@hotmail.com light perception after a bicycle crash. By funduscopy we found a peripapillary subretinal hemorrhage and a traumatic optic neuropathy was diagnosed. His visual acuity recovered just a few hours after the crash without special therapy and was 0,4 the next day. Two months later his visual acuity was 0,63 and the optic nerve showed signs of atrophy. Throughout the last years many studies and case series were published regarding high-dose steroid therapy in cases of traumatic optic neuropathy. There is a relatively high rate of spontaneous visual recovery and currently there is no convincing data that steroids provide any additional benefit over observation alone. An overview of these studies shows the controversial debate about high dose steroid therapy. Our case confirms that spontaneous partial recoveries occur.

Keywords Optic nerve injuries - Traumatic optic neuropathy $\cdot$ Steroids $\cdot$ Optic atrophy

\section{Anamnese}

Ein 34-jähriger Patient wurde um etwa 1 Uhr früh in unserer Notfallambulanz vorstellig. Er erzählte, am Nachhauseweg mit dem Fahrrad gestürzt zu sein, sich aber nicht mehr genau an das Unfallgeschehen erinnern zu können, da auch Alkohol im Spiel gewesen wäre. An Beschwerden gab er Kopfschmerzen und einen Visusverlust am linken Auge an. Eine unfallchirurgische Abklärung war bereits durchgeführt worden, hier konnte eine Nasenbeinfraktur und eine Orbitafraktur links aber ansonsten nur Prellungen festgestellt werden. Die ophthalmologische Anamnese war bisher unauffällig. 


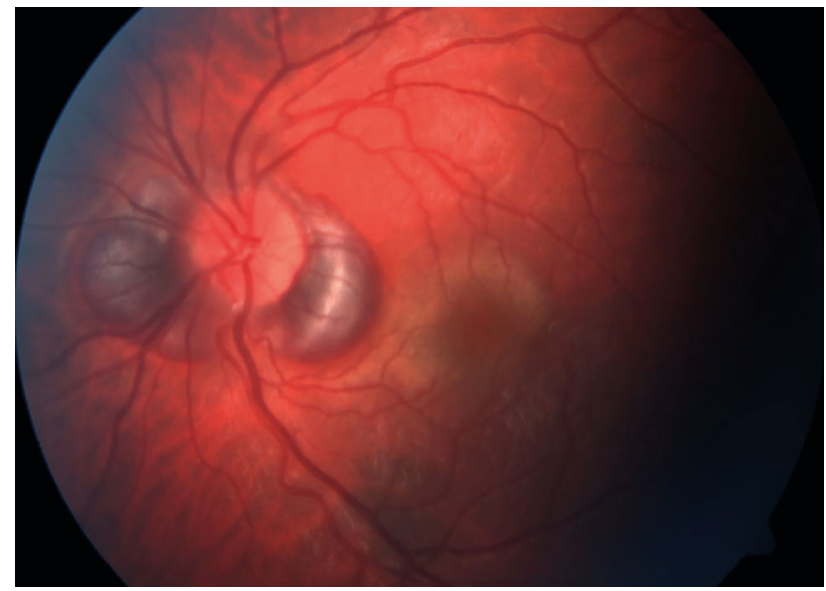

Abb. 1 Fundusbefund wenige Stunden nach dem Trauma. Es zeigt sich eine schmetterlingsförmige peripapilläre Blutung

\section{Befund}

Äußerlich fielen auf den ersten Blick multiple Schürfwunden am gesamten Gesichtsbereich auf. Der Fernvisus ergab rechts ohne Korrektur 0,8 und links keine Lichtwahrnehmung (KLE). Es zeigte sich ein relativer afferenter Pupillendefekt links. Der Augenvorderabschnitt präsentierte sich an der Spaltlampe unauffällig, abgesehen von wenig Blutfäden auf der Bindehaut. Funduskopisch konnte man peripapilläre, temporal und nasal (schmetterlingsförmig) ca. einen Papillendurchmesser große Blutungen erkennen aber kein Nervenfaserödem (Abb. 1).

Bei der genaueren Durchsicht der Computertomographie (CT) des Schädels fiel zusätzlich zur Impressionsfraktur des Orbitabodens und der medialen Wand der Orbita links auch eine lokalisierte Hypodensität des N. opticus auf. Es bestand eine diskrete schlierige Einblutung im dorsalen Abschnitt der Orbita ohne raumfordernden Effekt und der N. opticus erschien nicht bedrängt (Abb. 2).

\section{Diagnose}

Die erhobenen Befunde deuteten klar auf eine traumatische Optikusneuropathie (TON) hin. Es wird grundsätzlich zwischen einer direkten (z.B. penetrierende Fremdkörper oder Knochenfragmente, die den Nerv verletzen bis durchtrennen) oder indirekten (durch Scherkräfte) TON unterschieden. In den CT Bildern zeigte sich keine direkte Läsion des N. opticus, daher wurde eine indirekte TON diagnostiziert.

\section{Verlauf und Therapie}

Es erfolgte die stationäre Aufnahme an der Abteilung für Mund-, Kiefer- und Gesichtschirurgie zur antibiotischen Abschirmung, Schmerztherapie und Festlegen des weiteren operativen therapeutischen Procedere (die linke Orbita musste im Verlauf zwei
Wochen nach dem Unfallereignis operativ rekonstruiert und stabilisiert werden). Unsererseits wurden engmaschige ambulante/konsiliarische ophthalmologische Kontrollen durchgeführt. Von Seiten der kieferchirurgischen Kollegen wurde die Frage einer hochdosierten Steroidtherapie in den Raum gestellt, welche unsererseits jedoch nicht empfohlen und somit nicht durchgeführt wurde.

Erfreulicherweise zeigte sich bereits am Vormittag nur wenige Stunden nach dem Unfall ein Visusanstieg auf 0,4. Daher konnte auch ein Goldmann Gesichtsfeld durchgeführt werden in welchem am linken $\mathrm{Au}$ ge deutliche Einschränkungen oben, oben nasal und oben temporal zu sehen waren. Funduskopisch war die peripapilläre Blutung gleich geblieben und wurde mittels optischer Kohärenztomographie (OCT) dokumentiert und vermessen (Abb. 3 und 4). Es wurden auch visuell evozierte Potentiale abgeleitet, welche erwartungsgemäß im Vergleich zum rechten Auge deutlich reduzierte Amplituden zeigten.

Im weiteren Verlauf verbesserte sich der Visus auf 0,63 zwei Monate posttraumatisch. Die peripapilläre Blutung war $\mathrm{zu}$ diesem Zeitpunkt vollständig resorbiert aber die Papille deutlich abgeblasst. Der Gesichtsfelddefekt blieb nahezu gleich und auch der relative afferente Pupillendefekt war weiterhin vorhanden.

\section{Diskussion und Fazit für die Praxis}

Die TON ist ein eher seltener $(0,7 \%-2,5 \%)$ Grund für einen Visusverlust nach Schädeltrauma. Unser Patient wies die klassischen Merkmale auf: Es sind vor allem junge Männer betroffen (79-85 \%) und die Ursache des Traumas ist ein Fahrrad- oder Motorradsturz (49\%) [1].

Untypisch jedoch war die rasche und deutliche Visusverbesserung. Nicht einmal einen Tag später stieg er von KLE auf 0,4 an. Ein posttraumatischer Visus von KLE und das Vorhandensein einer Orbitafraktur sind prognostisch ungünstige Faktoren $[1,2]$. Weitere Faktoren, die ebenfalls einen schlechten Outcome des Visus prognostizieren wie Alter über 40 Jahre, Bewusstlosigkeit, Blutung in die hinteren Ethmoidalzellen und keine Visusverbesserung innerhalb von 48 Stunden [3] waren bei unserem Patienten nicht vorhanden.

Zur hochdosierten Kortisontherapie, die häufig bei TON angewendet wurde und immer noch in den Köpfen vieler vorhanden ist, sollte folgendes erörtert werden: Der vermehrte Einsatz dieser Therapie geht primär auf die National Acute Spinal Cord Injury Study zurück. Dies war eine randomisierte, doppelblinde, placebokontrollierte Multicenterstudie, die zeigte, dass Patienten mit Wirbelsäulenverletzungen, wenn sie innerhalb von acht Stunden mit hochdosierten intravenösem Kortison nach einem bestimmten Protokoll behandelt wurden, einen besseren Outcome hatten [4]. 
Abb. 2 SchädelCT bei Erstvorstellung am Tag des Traumas. Es zeigt sich eine unspezifische umschriebene Hypodensität des N. opticus noch im Bereich der Orbita aber keine direkte Läsion oder Unterbrechung in seinem Verlauf

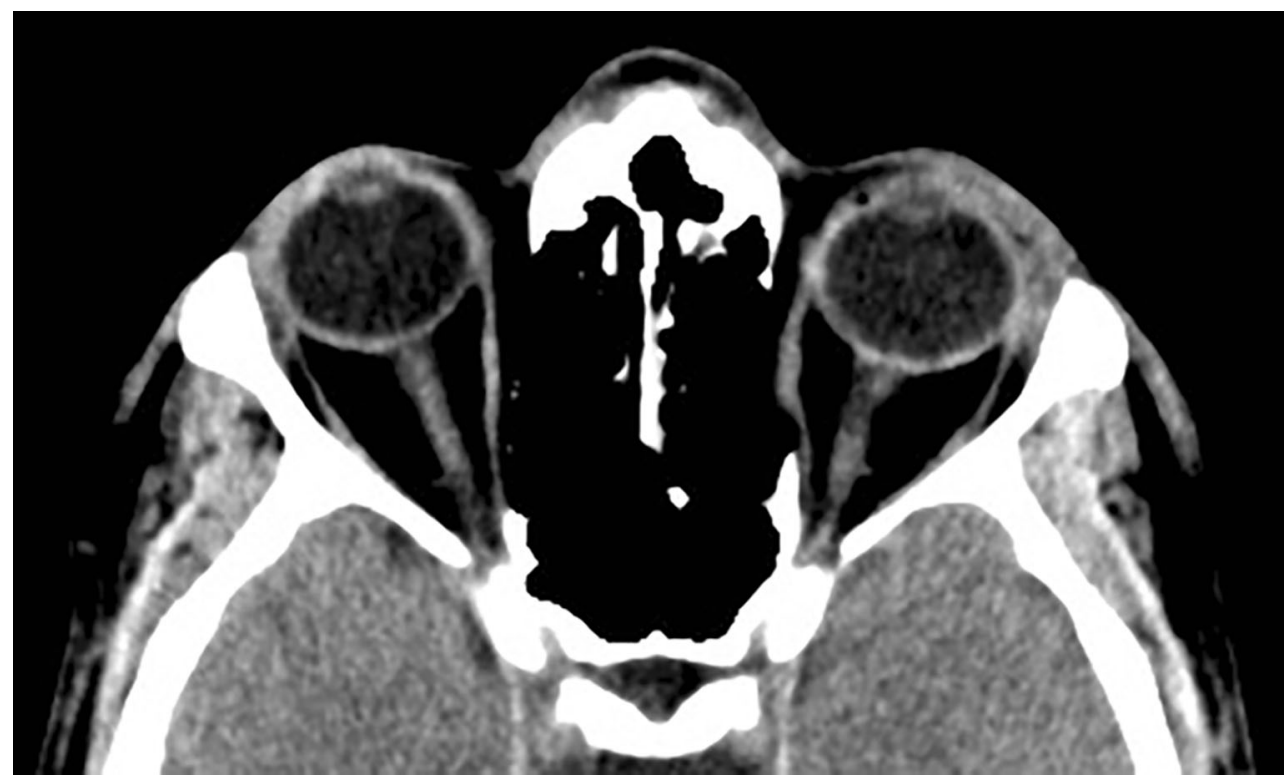

Abb. 3 OCTSchnitt quer durch diePapillemitder peripapillären subretinalen Blutung

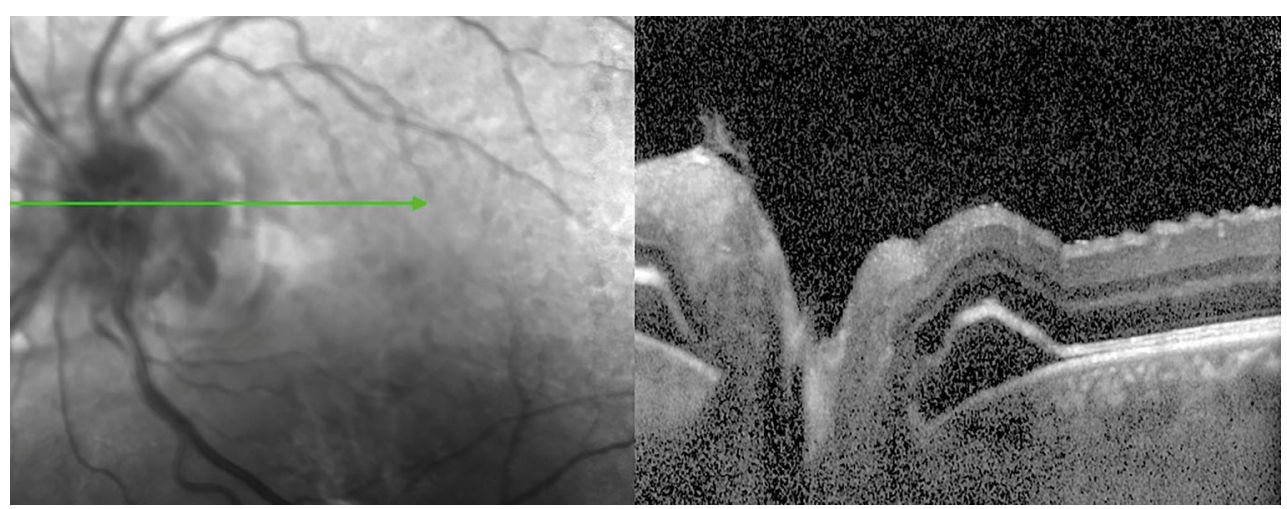

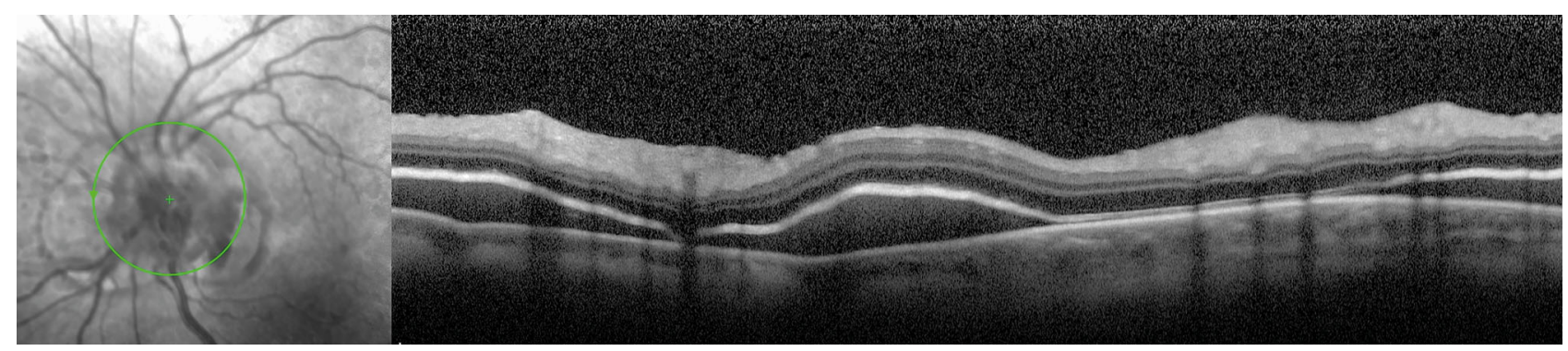

Abb. 4 Kreisrunder OCT Schnitt um die Papille mit der gut abbildbaren subretinalen Blutung

Seit 1990 haben zumindest 16 größere Studien/ Fallserien über die Therapie von TON mit Kortison berichtet. Keine dieser Studien hatte allerdings adäquate Kontrollgruppen, ja sogar die größte derartige Studie die International Optic Nerve Trauma Study [5] war eine nicht randomisierte, unkontrollierte Studie [6]. Sie zeigte, dass weder Dosis noch Zeitpunkt des Therapiebeginns von Kortison, noch die operative Dekompression des N. opticus einen Einfluss auf den Outcome des Visus im Vergleich zu keiner Therapie hatten [5]. Auch im Cochrane Review von 2013 konnte nur eine einzige randomisierte doppelblinde placebokontrollierte Studie gefunden werden [1]. In diese Studie wurden 31 Augen von 31 Patienten eingeschlossen und 16 davon mit hochdosiertem Kortison (in den ersten 3 Tagen $250 \mathrm{mg}$ Methylprednisolon alle 6 Stunden intravenös, danach niedriger dosiert und oral für weitere 11 Tage) und 15 erhielten als Placebo intravenöse Kochsalzlösung. Es ergab sich eine Visusverbesserung in $61,3 \%$ aller Patienten. Bei den mit Kortison therapierten Patienten verbesserte sich der Visus in $68,8 \%$, in der Placebo Gruppe in 53,3\% - ein statistisch nicht signifikanter Unterschied [7]. 
Abgesehen von Kortison sind in letzter Zeit auch neuere konservative Therapiestrategien untersucht worden. Eine unlängst publizierte Pilotstudie beschrieb den Effekt der intravenösen Erythropoietin (EPO) Gabe an 18 Patienten mit indirekter TON. EPO wird ein neuroprotektiver Effekt zugeschrieben. Bei dieser Fallserie ohne Kontrollgruppe zeigte sich eine signifikante Visusverbesserung, da die TON aber ohnehin eine hohe Spontanverbesserungsrate hat, sind hier für die richtige Beurteilung des Effektes randomisierte, kontrollierte Studien notwendig [8]. Noch in der (fernen?) Zukunft liegende, aber spannende neue Ansätze in der Therapie könnten auch in Form von Stammzellen liegen, die in Rattenbulbi injiziert die herbeigeführte Optikusneuropathie verbesserten [9].

Zusammenfassend kann gesagt werden, dass die Rate an Spontanheilung einer TON sehr hoch ist und es derzeit keine zuverlässigen Daten gibt, die einer Behandlung mit Kortison oder anderen möglicherweise neuroprotektiven Substanzen einen signifikanten Vorteil gegenüber der alleinigen Beobachtung zuschreiben. Es empfiehlt sich bei der derzeitigen Studienlage daher nicht nur „um zumindest irgendetwas zu tun“ eine Therapie, die bekanntermaßen selten auch schwerwiegende Nebenwirkungen nach sich ziehen könnte, zu versuchen [10].

Interessenkonflikt Sarah Krainz, Mona Regina Schneider, Bianca Bizjak und Andreas Wedrich geben an, dass kein Interessenkonflikt besteht.

Open Access This article is distributed under the terms of the Creative Commons Attribution 4.0 International License (http://creativecommons.org/licenses/by/4.0/), which permits unrestricted use, distribution, and reproduction in any medium, provided you give appropriate credit to the original author(s) and the source, provide a link to the Creative Commons license, and indicate if changes were made.

\section{Literatur}

1. Yu-Wai-Man P, Griffiths PG. Steroids for traumatic optic neuropathy. Cochrane Database Syst Rev. 2013;6:CD006032.

2. Wang BH, Robertson BC, Girotto JA, et al. Traumatic optic neuropathy: a review of 61 patients. Plast Reconstr Surg. 2001;107(7):1655-1664.

3. Carta A, Ferrigno L, Salvo M, Bianchi-Marzoli S, Boschi A, Carta F. Visual prognosis after indirect traumatic optic neuropathy. J Neurol Neurosurg Psychiatry. 2003;74(2):246-248.

4. Bracken MB, Shepard MJ, Collins WF, et al. A randomized, controlled trial of methylprednisolone or naloxone in the treatment of acute spinal-cord injury. results of the second national acute spinal cord injury study. N Engl J Med. 1990;322(20):1405-1411.

5. Levin LA, Beck RW, Joseph MP, Seiff S, Kraker R. The treatment of traumatic optic neuropathy: the international optic nerve trauma study. Ophthalmology. 1999;106(7):1268-1277.

6. Kenneth D, Steinsapir MD. Treatment of traumatic optic neuropathy with high-dose Corticosteroid. J Neuroophthalmol.2006;26:65-67.

7. Entezari M, Rajavi Z, Sedighi N, Daftarian N, Sanagoo M. High-dose intravenous methylprednisolone in recent traumatic optic neuropathy; a randomized double-masked placebo-controlled clinical tiral. Graefes Arch Clin Exp Ophthalmol.2007;245(9):1267-1271.

8. Entezari M, Esmaeili M, Yaseri M. A pilot study oft the effect of intravenous erythropoietin on improvement of visual function in patients with recent indirect traumatic optic neuropathy. Graefes Arch Clin Exp Ophthalmol. 2014;252:1309-1313.

9. Zhu Q, Liu Z, Wang C, et al. Lentiviral-mediated growthassociated protein- 43 modification of bone marrow mesenchymal stem cells improves traumatic optic neuropathy in rats. Mol Med Rep. 2015;12(4):5691-5700.

10. Chaon BC, Lee MS. Is there treatment for traumatic optic neuropathy? Curr Opin Ophthalmol. 2015;26(6):445-449. 